\title{
Aquaporin 1 plays an important role in myocardial edema caused by cardiopulmonary bypass surgery in goat
}

\author{
YUMEI YAN ${ }^{*}$, JIANBIN HUANG* , FANGBAO DING, JU MEI, JIAQUAN ZHU, HAO LIU and KUN SUN \\ Department of Cardiothoracic Surgery, Xinhua Hospital, School of Medicine, \\ Shanghai Jiaotong University, Shanghai 200092, P.R. China
}

Received October 16, 2012; Accepted December 3, 2012

DOI: $10.3892 /$ ijmm.2013.1228

\begin{abstract}
Myocardial stunning, which is closely related to myocardial edema, is a severe complication that may occur following cardiac surgery. In this study, we examined the expression of aquaporin 1 (AQP1) and Connexin 43 (Cx43) following cardiopulmonary bypass $(\mathrm{CPB})$ surgery in goats. We assessed myocardial muscle tissue water content according to changes in dry-wet weight. Our results showed that AQP1 expression and myocardial muscle tissue water content increased significantly $6 \mathrm{~h}$ after CPB surgery, reaching peak levels $48 \mathrm{~h}$ after surgery; additionally, the protein expression of Cx43 was inversely correlated with AQP1 expression. Overexpression of AQP1 during CPB surgery enhanced the degree of myocardial edema, whereas the addition of water channel protein inhibitor $\mathrm{Hg}^{2+}$ in cold crystalloid cardioplegia and knockdown of AQP1 during surgery weakened the degree of myocardial edema. These findings revealed that the severity of myocardial edema after CPB surgery is correlated with AQP1 protein expression levels, suggesting the important role played by AQP1 protein in the regulation of $\mathrm{Cx} 43$ in the pathological progression of myocardial edema.
\end{abstract}

\section{Introduction}

Myocardial edema is closely related to myocardial stunning, and may lead to death or complications following surgery. Heart dysfunction caused by myocardial edema can dramatically affect both systolic and diastolic function, and may endure over a long period of time $(1,2)$. It has been previously demonstrated that a water content increase of $3.5 \%$ can lead to a cardiac output decrease of 30 to $50 \%(3,4)$. Myocardial

Correspondence to: Dr Fangbao Ding, Department of Cardiothoracic Surgery, Xinhua Hospital, School of Medicine, Shanghai Jiaotong University, 1665 Kongjiang Road, Shanghai 200092, P.R. China

E-mail: drnail@126.com

*Contributed equally

Key words: myocardial edema, aquaporin 1, water channel, extracorporeal circulation, Connexin 43 edema is caused by ischemia-reperfusion injury, hemodilution during cardiopulmonary bypass (CPB), and an inflammatory response to $\mathrm{CPB}(5,6)$.

Aquaporin water channels are comprised of highly conserved proteins that exist in both bacteria and humans. These water channels are involved in many physiological processes, such as the osmotic environment and overall body fluid balance, including osmotically driven transepithelial fluid transport that occurs in the kidney, eye and secretory glands, and facilitation of water movement into and out of the brain in various pathologies such as stroke, tumor and infection (7-10). There are 13 members of AQPs (AQP0-AQP12) in animals and their expression is organ-specific. Aquaporin 1 (AQP1) is the most predominant and least tissue expressionspecific subtype. The monomeric structure consists of two triple transmembrane helices connected by a long flexible extracellular loop. Studies in rodents have demonstrated that AQP1 is expressed in cardiac muscle cells and that it can be reversibly internalized from the cell membrane in response to the osmotic environment (10-12).

Cardiac myocytes are tightly interconnected by means of highly specialized regions of the plasma membrane called gap junctions. Gap junctions are composed of clusters of transmembrane channels connecting the cytosol of adjacent cells. Studies have demonstrated that Connexin $43(\mathrm{Cx} 43)$ plays an important role in myocardial protection in ischemic preconditioning (13-15), but whether it plays the same role in myocardial edema remains unknown.

In this study, we demonstrated the variations in mRNA expression and protein levels of AQP1 following CPB surgery in a goat animal model. To maintain constant extracellular volume, water is cleared from the cytoplasm via gap junctions. We also tested whether a functional relationship exists between water channels and gap junctions. We found that AQP1 plays an important role in myocardial edema via regulation of $\mathrm{Cx} 43$ expression.

\section{Materials and methods}

Animal model. The study protocol was approved by the Medical Ethics Committee of Shanghai Xinhua Hospital, conforms to the Principles of Laboratory Animal Care (National Society for Medical Research), and was conducted according to National Institutes of Health guidelines. 
Twenty-four adult goats weighing 50-60 kg (Slac Laboratory Animal) were used for this study. Myocardium tissues from the left and right cardiac ventricles were obtained from six adult goats anesthetized and bled from the dorsal aorta until expiration. Tissues were stored in liquid nitrogen for further use.

Animal studies and extracorporeal circulation model. The goats underwent general anesthesia with isoflurane and nitrous oxide, and were endotracheally intubated. A left anterolateral thoracotomy was performed in the fifth intercostal space, followed by dissection. After systemic anticoagulation with sodium heparin $(300 \mathrm{U} / \mathrm{kg})$, the aorta was cannulated for arterial perfusion. A venous cannula was placed in the right atrium. Upon initiation of CPB surgery, the body temperature was cooled to $28^{\circ} \mathrm{C}$ and the aorta was cross-clamped. The heart was arrested with crystalloid cardioplegic solution.

The pericardial cavity was filled with cold physiological saline after cardiac arrest and perfused with crystalloid cardioplegic solution every $20 \mathrm{~min}$. Following $60 \mathrm{~min}$ of cardiac arrest, the aorta was declamped. All animals were weaned from CPB without inotropic support $60 \mathrm{~min}$ after the release of the aortic cross-clamp. Protamine was used to neutralize the heparin. Following placement of the drainage tube, the thoracic cavity was closed.

Myocardium tissue samples from the left and right cardiac ventricles were obtained $0,2,6,12,24,48$ and $72 \mathrm{~h}$ following $\mathrm{CPB}$, and stored in liquid nitrogen until further use.

Real-time PCR analysis of AQP1 expression. Real-time PCR was performed to assess AQP1 expression in the left ventricle (LV) and right ventricle (RV). Heart tissue was homogenized and RNA was isolated using RNeasy Fibrous Tissue Mini kit (Qiagen) according to the manufacturer's protocol, and any contaminating DNA was degraded by a $15-\mathrm{min}$ incubation with RNase-free DNase. Real-time PCR was performed using the High Capacity cDNA Archive kit (Applied Biosystems). We created two standard curves, one for mouse AQP1 and the other using the internal control GAPDH with real-time PCR using appropriate primers and probes (Applied Biosystems). Each sample was loaded in triplicate for both AQP1 and GAPDH. The primers for AQP1 were: forward, 5'-GCC AGC GAG TTC AAG AAG-3' and reverse, 5'-CCC CAC CCA GAA AAT CC-3'. The primers for GAPDH were: forward, 5'-GCC AGC GAG TTC AAG AAG-3' and reverse, 5'-CCC CAC CCA GAA AAT CC-3'. Real-time PCR was conducted using the ABI Prism 7000 sequence detection system, and data were analyzed with ABI Prism 7300 SDS software.

Western blot analysis. Western blot analysis was performed as described previously (16), with minor modifications. In brief, each goat myocardium tissue sample was crushed and ground using a mortar, pestle and liquid nitrogen. The resulting powder was immediately suspended in lysis buffer $(\mathrm{pH} \mathrm{7.4,}$ 4\% SDS, $100 \mathrm{mM}$ DTT, $125 \mathrm{mM}$ Tris, $40 \%$ glycerol and trace cocktail protease inhibitor), and ultrasonically homogenized. Next, the solution was heated to $94^{\circ} \mathrm{C}$ for $4 \mathrm{~min}$, and the cell debris and insoluble substances were removed by centrifugation at $15,000 \times \mathrm{g}$ for $3 \mathrm{~min}$. The supernatant was separated by SDS-PAGE with $20 \mu \mathrm{g}$ protein/lane and transferred onto a
PVDF membrane (Millipore) using transfer buffer [pH 11.0, $25 \mathrm{mM}$ Tris, $0.2 \mathrm{M}$ glycine, $20 \%$ (v/v) methanol] on a semi-dry electroblotter (Bio-Rad). After blocking with 5\% skim milk and $0.1 \%$ Tween-20 for $1 \mathrm{~h}$ at room temperature (RT), AQP1 was detected by incubating the samples with rabbit polyclonal anti-AQP1 and Cx43 antibodies at a 1:1,000 dilution (Abcam Ltd.) overnight at $4{ }^{\circ} \mathrm{C}$. Alkaline phosphatase-goat anti-rabbit IgG at a 1:3,000 dilution (GeneTex, Inc., USA) was used as the secondary antibody. The immunoblots were developed using ECL detection reagent (Pierce Chemical).

Lentivirus production and transfection. We obtained the lentivirus packaging system for AQP1 overexpression from Tronolab. The lentivirus packaging system for AQP1 RNAi was obtained from Addgene. The RNAi target sequences were 5'-ATC ATC AGC ATC CAA GGT CAT ACT CC-3' and 5'-AAG AGC TTC TTC TTG ATT TCG CTG G-3'. Recombinant lentivirus was produced by co-transfecting $293 \mathrm{~T}$ cells with the expression plasmid and packaging plasmids, and RNAi plasmids and packaging plasmids separately. Infectious cells were harvested at 48 and $72 \mathrm{~h}$ post transfection. Cell debris was eliminated by centrifugation. Recombinant lentiviral suspension ( $200 \mu \mathrm{g} / \mathrm{kg}$ body weight) was injected into the ventricular myocardium of the ventricular wall using 30-gauge syringe. The injection did not cause any hemodynamic changes, allergies, or other side effects during the entire experiment. The empty vector virus was injected in an identical manner and served as the negative control.

Immunofluorescence. Cryosections (8- $\mu \mathrm{m})$ were fixed with $3 \%$ paraformaldehyde in PBS, washed, and incubated in blocking buffer (PBS containing 2\% BSA). Primary antibodies diluted in blocking buffer were applied for $1 \mathrm{~h}$ at RT, or overnight at $4{ }^{\circ} \mathrm{C}(1: 1,000$ dilutions for the monoclonal AQP1 antibody; Abcam). Samples were washed with PBS and incubated for $2 \mathrm{~h}$ with appropriate secondary antibodies diluted in blocking buffer (Alexa Fluor 488-conjugated donkey anti-rabbit IgG; Molecular Probes-Invitrogen). Samples were washed three times in PBS and mounted using Shandon Immu-mount ${ }^{\mathrm{TM}}$. Sections were analyzed with a Leica SP2 confocal laserscanning microscope.

Analysis of myocardial edema. Myocardial edema was determined according to the water content of the myocardial muscle tissue. The wet weight (WW) of myocardial tissue was weighed using an analytical balance. The samples were then dried using a microwave oven, and the dry weight (DW) was recorded. Water content was calculated using the equation: Water content $=(\mathrm{WW}-\mathrm{DW}) / \mathrm{WW} \times 100 \%$.

Cardiac function measurement. A micromanometer tipped catheter was positioned in the LV. Hemodynamic parameters were recorded using a data recording unit. Echocardiography was performed using a Sonos 5500 (Hewlett Packard, USA).

Statistical analysis. All values are expressed as the mean \pm standard deviation (SD). Statistical analysis was performed by one-way analysis of variance (ANOVA), followed by the Student's and Newman-Keuls test using SPSS 11.0. A P-value $<0.05$ was considered to indicate a statistically significant result. 

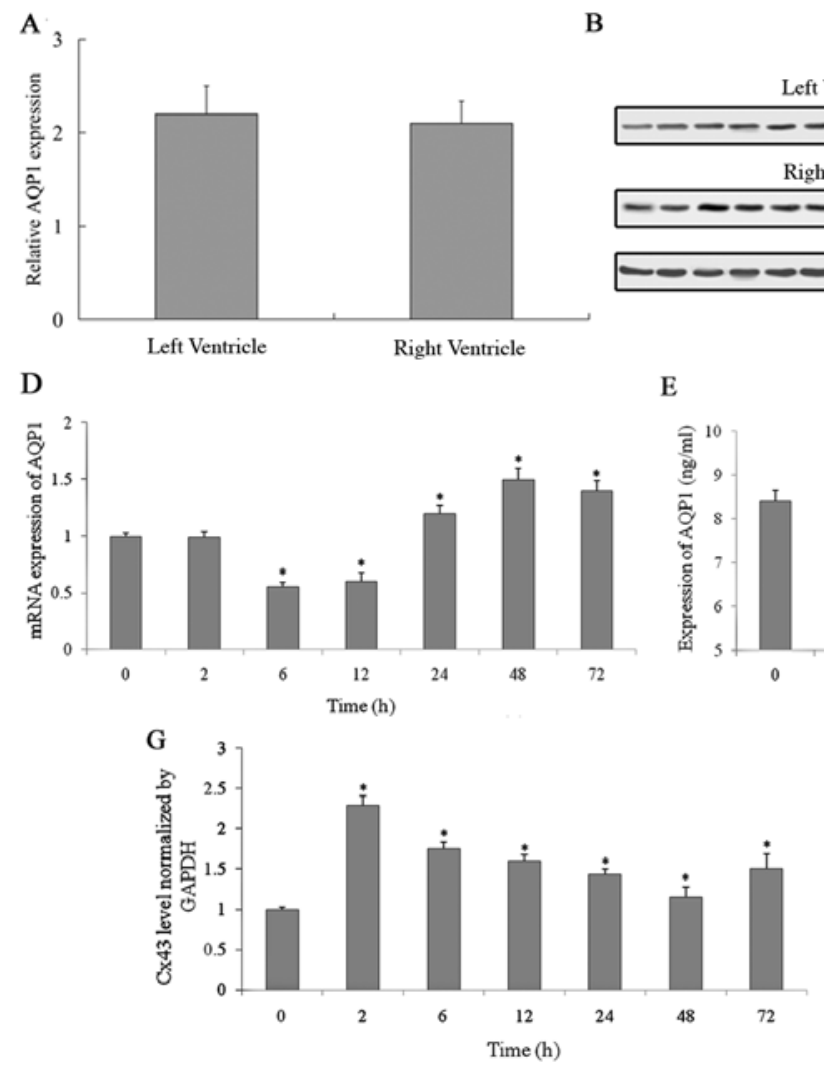

B

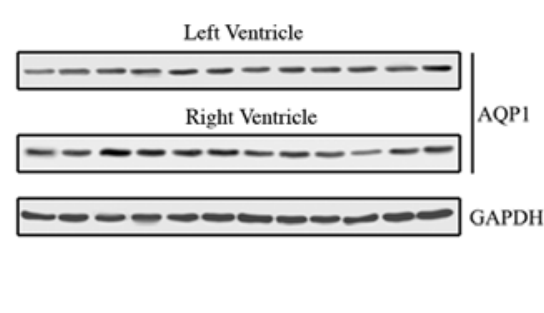

$\mathrm{E}$

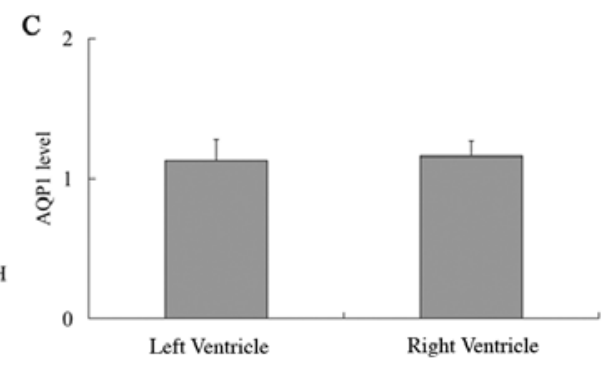

F
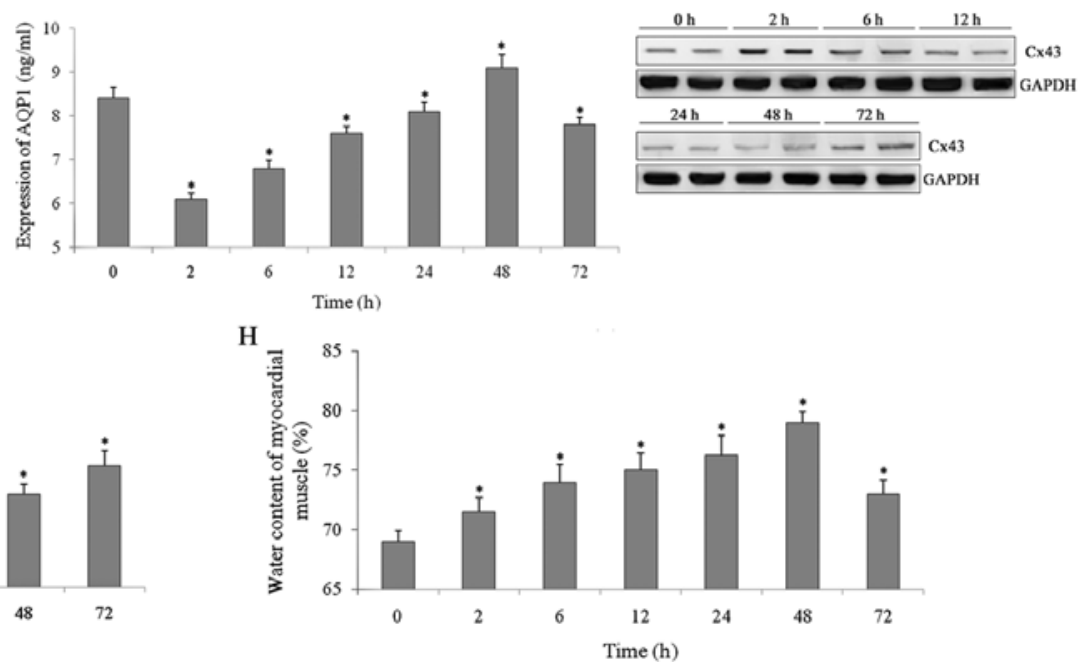

Figure 1. mRNA or protein expression of AQP1 and Cx43 in the left and right ventricles of goats. Samples (from six left and six right ventricles) were obtained from $40 \mathrm{~kg}$ adult goats (3 males and 3 females). (A) mRNA expression were detected by real-time PCR; each sample was repeated three times. Error bars represent standard deviation $(n=6)$. (B) Protein expression was detected by western blot analysis; each sample was repeated two times using GAPDH as the control. (C) Histogram based on the gray values of the western blot analysis; error bars represent standard deviation (n=6). (D) mRNA expression levels of AQP1 analyzed by real-time PCR at different time-points after cardiopulmonary bypass (CPB) surgery; error bars represent standard deviation ( $\mathrm{n}=6$ ). (E) Protein expression levels of AQP1 were analyzed by ELISA at different time-points after CPB surgery, error bars represent standard deviation ( $\mathrm{n}=6$ ). (F) Protein expression levels of Cx43 were analyzed by western blot analysis at different time-points after CPB surgery (n=6). (G) Quantification of Cx43 protein levels from western blot analysis; data were normalized by corresponding GAPDH. * $\mathrm{P}<0.05$. (H) The water content of myocardial muscle tissue at different time-points after CPB surgery; error bars represent standard deviation $(\mathrm{n}=6)$. ${ }^{*} \mathrm{P}<0.05$ vs. 0 h following $\mathrm{CPB}$ surgery.

\section{Results}

Right and left ventricular expression of AQP1 in goats. The expression of AQP1 in human cardiac muscle is rather high. In our study, goat right and left ventricular mRNA expression levels of AQP1 were detected by real-time PCR, and the results indicated that there was little difference between the right and the left ventricles $(\mathrm{P}>0.05)$ (Fig. 1A). The protein levels of AQP1 were also detected by western blot analysis (Fig. 1B). A histogram was created based on the gray values of the western blot analysis (Fig. 1C). The protein level of AQP1 in the left and right ventricles was also nearly identical $(\mathrm{P}>0.05)$ (Fig. 1B and C).

Left ventricular expression of $A Q P 1$ and $C x 43$ after $C P B$ surgery. The mRNA expression of AQP1 was measured by real-time-PCR $0,2,6,12,24,48$ and $72 \mathrm{~h}$ following CPB surgery (Fig. 1D). Initially, mRNA expression decreased after aortic occlusion. Six hours later, the expression began to increase and reached a maximum level after $48 \mathrm{~h}$. Next, the mRNA expression level of AQP1 slowly decreased. The protein expression of AQP1 was measured using ELISA (Fig. 1E), and the expression pattern was similar to mRNA expression. Initially the protein expression decreased, $6 \mathrm{~h}$ later the expression level began to increase, $48 \mathrm{~h}$ later it reached the peak value, and then the expression level began to slowly decrease. The protein expression of $\mathrm{Cx} 43$ (Fig. 1F) detected by western blot analysis, was inversely correlated with AQP1.

Myocardial edema was assessed according to the water content of the myocardial muscle. The water content of the myocardial muscle was measured 2, 6, 12, 24, 48 and $72 \mathrm{~h}$ following CPB surgery (Fig. $1 \mathrm{H}$ ). The degree of myocardial edema increased following the cardiopulmonary surgery, and $48 \mathrm{~h}$ later peaked at the same time that the mRNA and protein expression levels of AQP1 reached maximum values. Finally, the water content of the myocardial muscle slowly decreased.

Lentiviral-mediated overexpression of AQP1 causes myocardial edema. Left and right ventricular cells were infected by a lentivirus expressing AQP1 during CPB surgery. The expression of AQP1, as detected by real-time PCR (Fig. 2A-D), and the protein expression of $\mathrm{Cx} 43$, detected by western blot analysis (Fig. 2G-H), decreased after infection with the lentivirus expressing AQP1. A similar trend was observed between AQP1 expression levels and the control; however, the quantity of AQP1 protein expression was greater compared to the control (Fig. 2B and D). Myocardial edema was determined by measuring the water content of the myocardial muscle tissue 
A
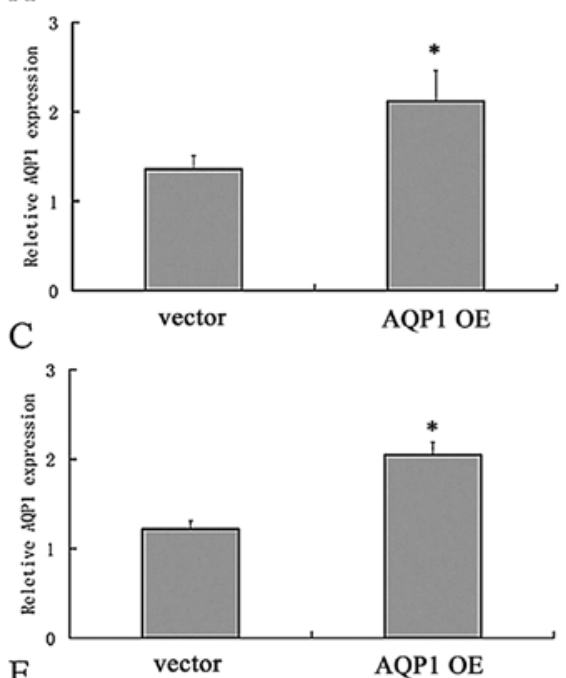

$\mathrm{E}$

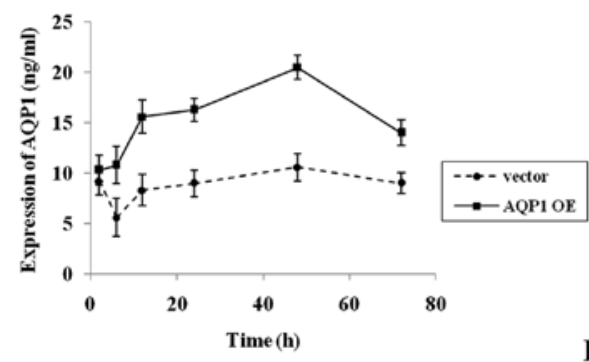

B
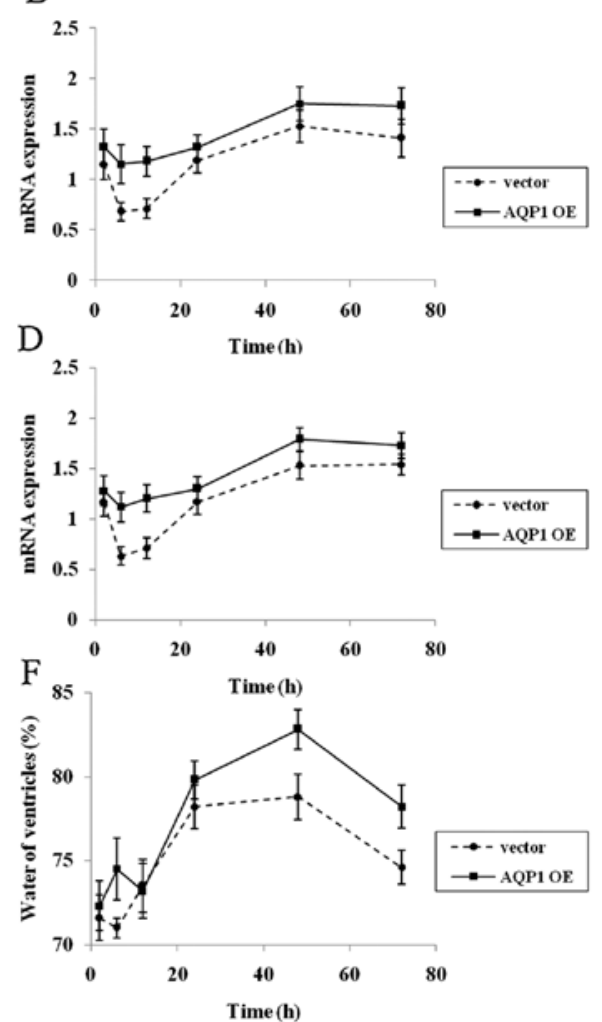

$\mathrm{H}$

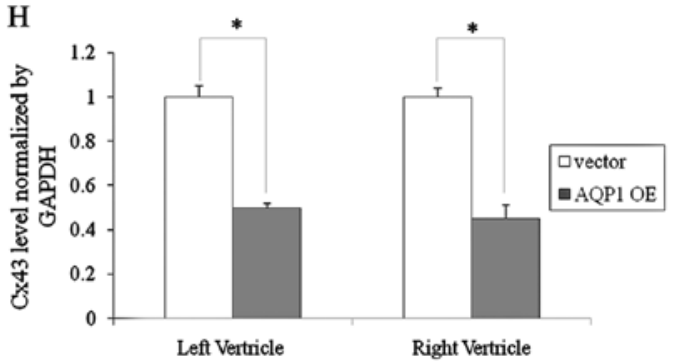

Figure 2. Expression of AQP1 and Cx43 after lentivirus infection. (A) Relative mRNA expression of AQP1 in the left ventricle 48 h post-lentivirus infection. (B) Relative mRNA expression of AQP1 in the left ventricle after lentivirus infection during cardiopulmonary bypass (CPB) surgery. (C) Relative mRNA expression of AQP1 in the right ventricle $48 \mathrm{~h}$ post-lentivirus infection. (D) Relative mRNA expression of AQP1 in right ventricle post-lentivirus infection during CPB surgery. (E) Protein expression levels of AQP1 in right ventricle post-lentivirus infection during CPB surgery according to ELISA analysis. (F) Water content of myocardial muscle tissue post-lentivirus infection during CPB surgery. Error bars represent standard deviation ( $\mathrm{n}=6$ ). $(\mathrm{G})$ Protein expression levels of $\mathrm{Cx} 43$ in the left and right ventricle $48 \mathrm{~h}$ post-lentivirus infection during CPB surgery analyzed by western blot analysis. (H) Quantification of Cx43 protein levels according to western blot analysis; data were normalized by corresponding GAPDH. * P<0.05 vs. empty vector group. Vector, empty vector group; AQP1 OE, AQP1 overexpression group.

(Fig. 2F). The myocardial edema of the infection group was much greater compared to the control group. The changes of AQP1 protein over time were also measured (Fig. 2E). The trend of the curve correlated with the degree of myocardial edema.

Inflammation was observed in conjunction with elevated $A Q P 1$ expression following $C P B$ surgery. AQP1 was knocked down following injection of an RNAi lentivirus during CPB surgery. Tissue samples were obtained from the left ventricle. Sections were cut $(5 \mu \mathrm{m})$ and stained using the standard hematoxylin and eosin staining. The efficiency of knockdown was detected by immunofluorescence staining (Fig. 3B). In the control group, a greater number of nuclei was released from the myocardial cells (Fig. 3A). This finding revealed that inflammation had occurred along with myocardial edema following CPB surgery. Inflammation did not occur in the normal tissue group or in the surgery with lentivirus-infection group. The expression of $\mathrm{Cx} 43$ (Fig. 3C) increased in the surgery with lentivirus-infection group compared to the control group; this demonstrated that the inflammation caused by myocardial edema correlated with AQP1 and Cx43 expression.

Effect of water channel protein inhibitor $\mathrm{Hg}^{2+}$ on myocardial edema caused by $C P B . \mathrm{Hg}^{2+}$ is a water channel protein inhibitor. During CPB surgery, $3 \mu \mathrm{M} \mathrm{Hg}^{2+}$ was added to cardioplegia. The mRNA and protein expression levels of AQP1 were measured 2, 6, 12, 24, 48 and $72 \mathrm{~h}$ following CPB surgery (Fig. 4A-D). The protein expression of $\mathrm{Cx} 43$ was measured $48 \mathrm{~h}$ after $\mathrm{CPB}$ surgery (Fig. 4E and F). Our results revealed that $\mathrm{Hg}^{2+}$ exhibited no effect on both the left and right ventricular expression of AQP1 ( $>>0.05)$. However, the degree of myocardial edema was low compared with the control group. 
A

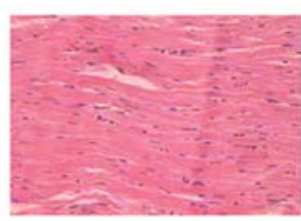

Normal tissue

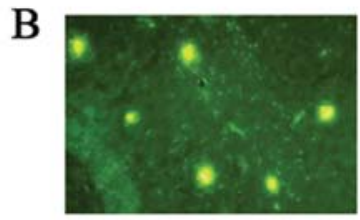

Normal tissue

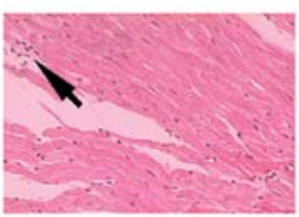

Sham control

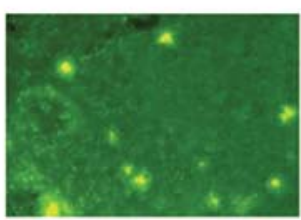

Sham control

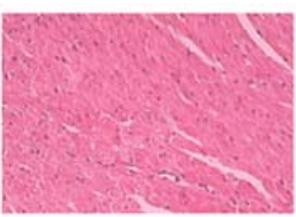

AQP1 RNAi

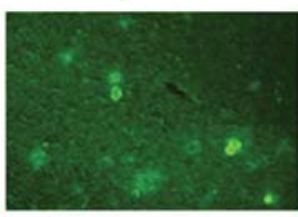

AQP1 RNAi

$\mathrm{C}$

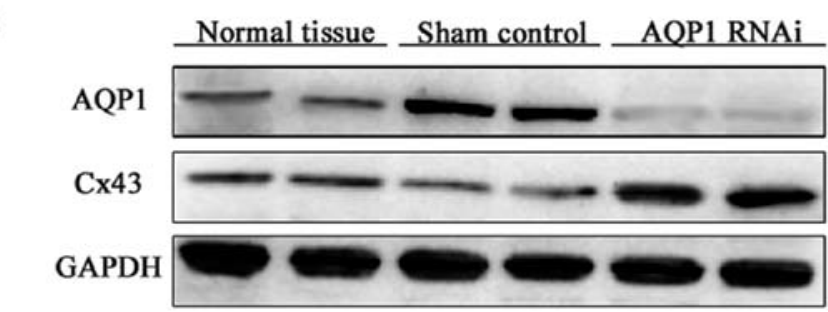

D

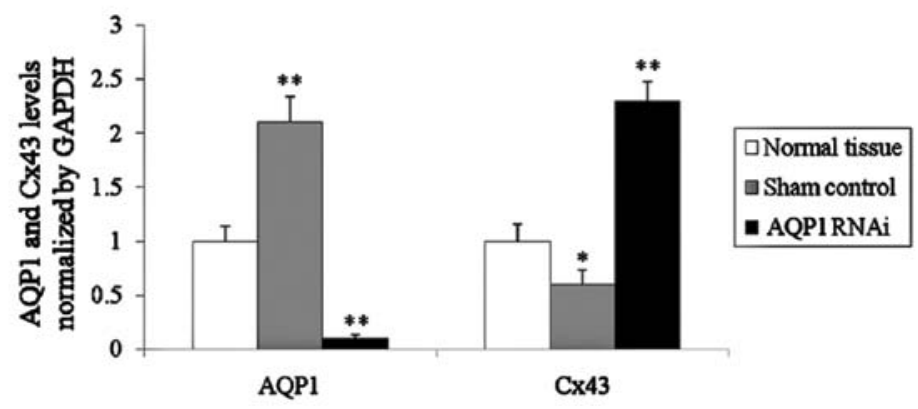

Figure 3. Tissue sections $48 \mathrm{~h}$ after injection of AQP1 RNAi following cardiopulmonary bypass (CPB) surgery and immunolocalization of AQP1. (A) H\&E staining of cardiac muscle sections. (B) Detection of AQP1 by immunofluorescence. Compared with normal tissue and tissue sections infected with AQP1 RNAi, there were a greater number of nuclei (arrows) released from cells in the sham control (surgery without lentivirus infection). (C) Protein expression levels of AQP1 and Cx43 were measured by western blot analysis. (D) Quantification of AQP1 and Cx43 protein levels according to western blot analysis; data were normalized by corresponding GAPDH. ${ }^{*} \mathrm{P}<0.05,{ }^{* *} \mathrm{P}<0.01$ compared to the normal tissue group.

The effect of lentiviral-mediated overexpression of $A Q P 1$ on cardiac function following CPB surgery. To confirm the effects of lentiviral-mediated overexpression of AQP1 in myocardial edema, lentiviral vector of AQP1 or empty vector was injected intramuscularly into goats during CPB surgery; cardiac function was then assessed using hemodynamic parameters and catheterization analysis 2, 6, 12, 24, 48 and $72 \mathrm{~h}$ following CPB surgery. Catheterization analysis revealed the LV pressure (LVP) of AQP1 lentiviral vector- and empty vector-treated goats (Fig. 4H). In both groups, the LVP gradually recovered following CPB surgery; however, at the $72 \mathrm{~h}$ time-point, the LVP of the AQP1 lentiviral vector-treated goats was significantly lower compared to the empty vector-treated goats (AQP1 lentiviral vector, $76.12 \pm 7 \mathrm{mmHg}$; empty vector, $98.03 \pm 10 \mathrm{mmHg}$; $\mathrm{P}<0.05)$. Positive and negative $\mathrm{dP} / \mathrm{dt}$ was used to measure the overall cardiac contractility and relaxation, respectively (17). Positive $\mathrm{dP} / \mathrm{dt}$ decreased following CPB surgery in the empty vector- and AQP1 lentiviral vector-treated goats (Fig. 4I). The positive $\mathrm{dP} / \mathrm{dt}$ of the AQP1 lentiviral vector-treated goats was significantly lower compared to empty vector-treated goats $72 \mathrm{~h}$ after CPB surgery (AQP1 lentiviral vector, $822 \pm 59 \mathrm{mmHg}$; empty vector, $965 \pm 72 \mathrm{mmHg} ; \mathrm{P}<0.05)$. Moreover, recovery of negative $\mathrm{dP} / \mathrm{dt}$ after $\mathrm{CPB}$ surgery was observed in the empty vector- and AQP1 lentiviral vector-treated goats (Fig. 4J). The negative $\mathrm{dP} / \mathrm{dt}$ of AQP1 lentiviral vector-treated goats was significantly worse compared to empty vector goats $24 \mathrm{~h}$ after CPB surgery (AQP1 lentiviral vector, $-1150 \pm 126 \mathrm{mmHg}$; empty vector, $-1432 \pm 97 \mathrm{mmHg} ; \mathrm{P}<0.05)$.

\section{Discussion}

This study was designed to examine the role of AQP1 in myocardial edema caused by CPB surgery. Additionally, we investigated whether there is a functional relationship between water channels and gap junctions. Using a goat extracorporeal circulation model, we examined APQ1 and Cx43 expression and the degree of myocardial edema during $\mathrm{CPB}$ surgery. We found a correlation between increased expression of AQP1 and myocardial edema following CPB surgery. Additionally, overexpression of AQP1 by a lentivirus enhanced myocardial edema and downregulated $\mathrm{Cx} 43$ expression.

AQP1 is the ubiquitous water channel protein found in endothelial cell membranes of vascular tissues throughout the body. It is found in the plasma membranes of red blood cells 

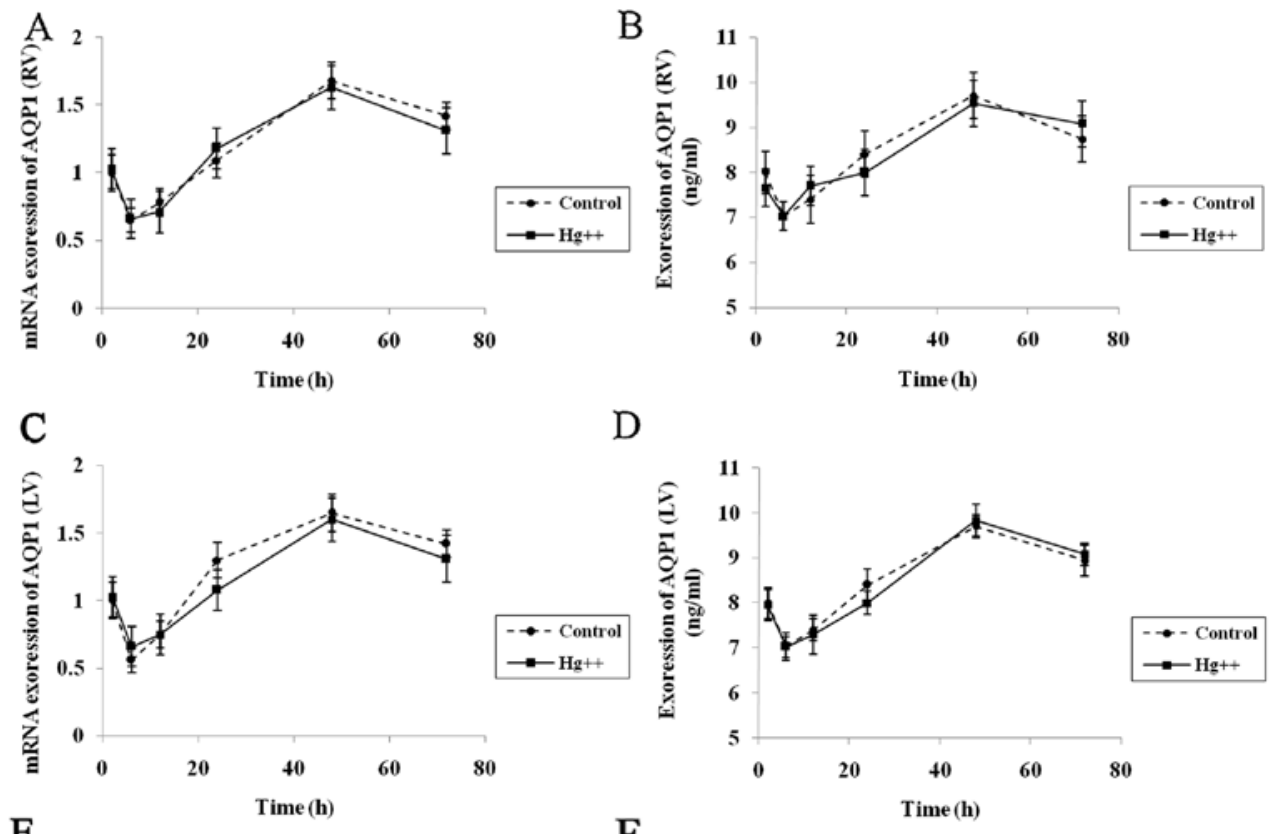

$\mathrm{E}$

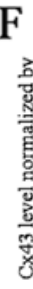
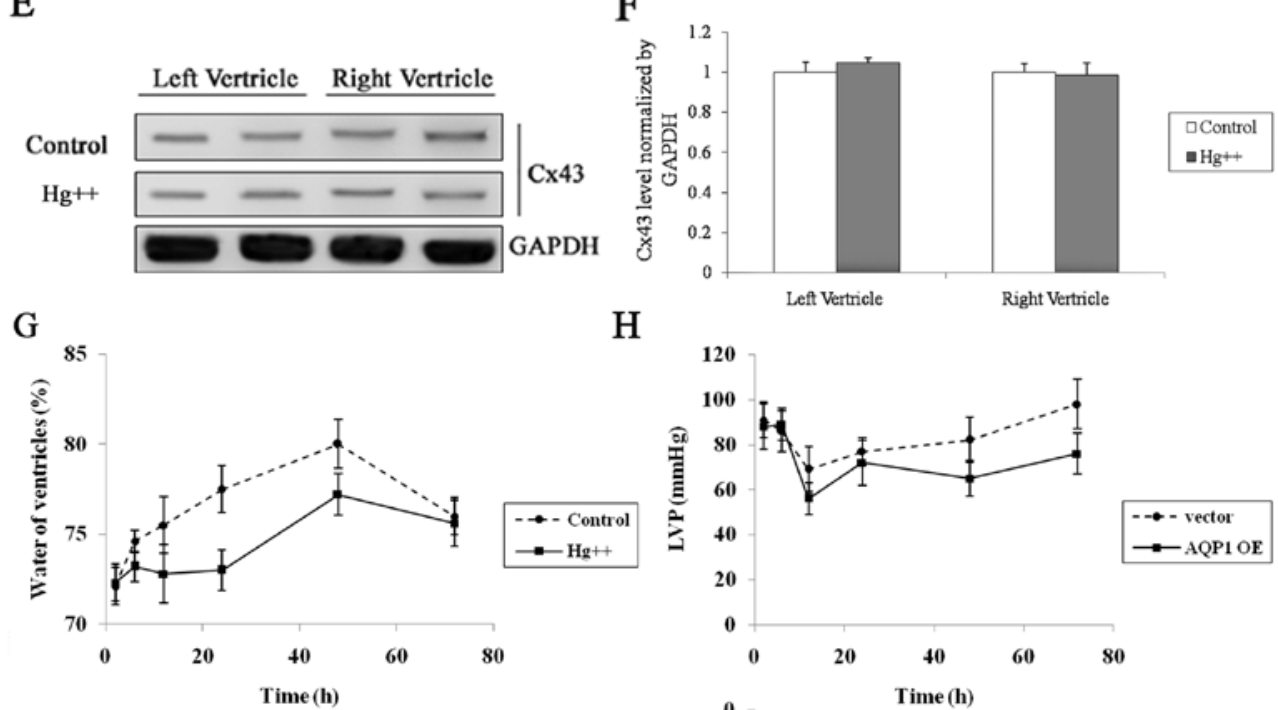

$\mathrm{H}$
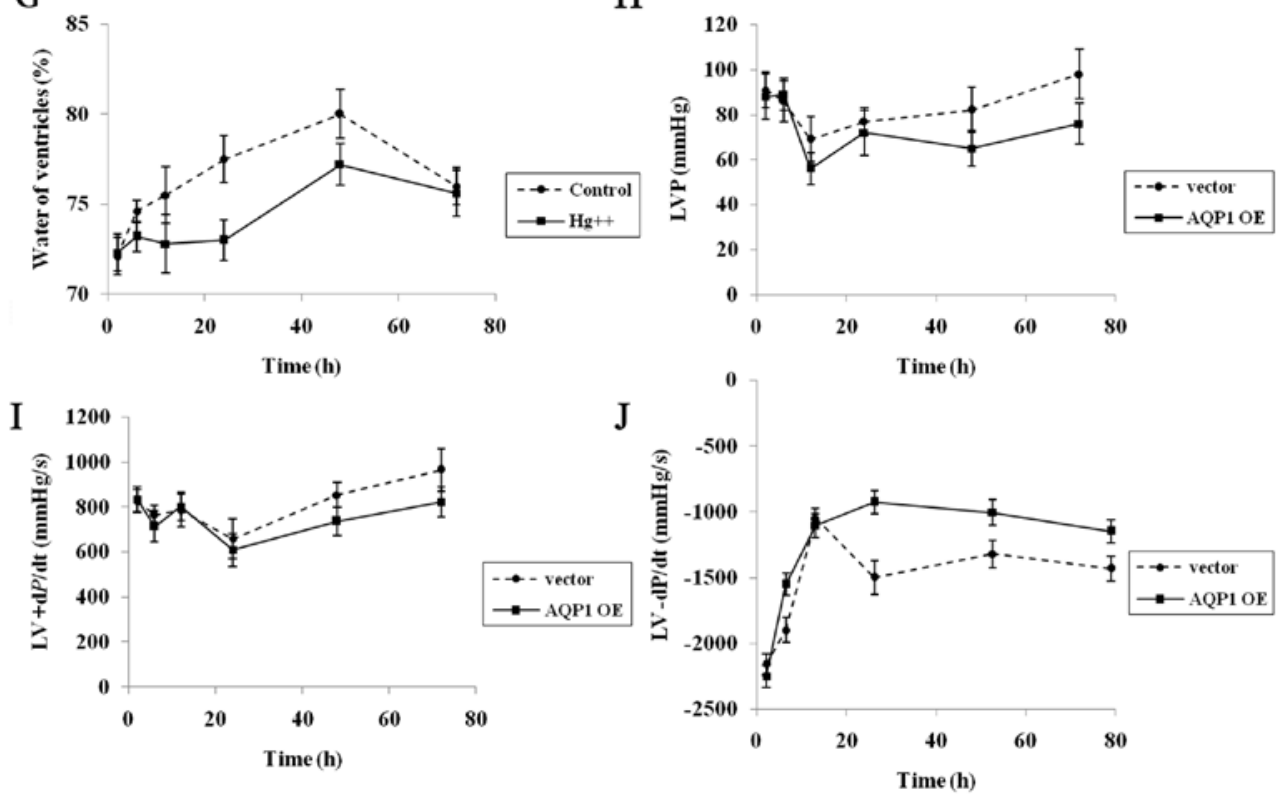

Figure 4. Expression of $\mathrm{AQP1}$ after $\mathrm{Hg}^{2+}$ treatment and during cardiopulmonary bypass (CPB) surgery and the effects on cardiac function after lentivirus infection. (A) Left ventricular and (C) right ventricular AQP1 mRNA expression levels after $\mathrm{Hg}^{2+}$ treatment during CPB surgery. (B) Left ventricular and (D) right ventricular $\mathrm{AQP1}$ protein expression after $\mathrm{Hg}^{2+}$ treatment during $\mathrm{CPB}$ surgery. (E) Protein expression levels of $\mathrm{Cx} 43$ in left and right ventricles $48 \mathrm{~h}$ after $\mathrm{Hg}^{2+}$ treatment following CPB surgery. (F) Quantification of $\mathrm{Cx} 43$ protein levels according to western blot analysis; data were normalized by corresponding GAPDH. (G) Water content of myocardial muscle tissue after $\mathrm{Hg}^{2+}$ treatment during CPB surgery. (H) Representative LVP records, (I) representative positive $\mathrm{dP} / \mathrm{dt}$ and $(\mathrm{J})$ representative negative $\mathrm{dP} / \mathrm{dt}$ of vector and AQP1 OE groups. Vector, empty vector group; AQP1 OE, AQP1 overexpression group.

as well as the kidney, lung, brain, and eye (18). Gap junctions are composed primarily of $\mathrm{Cx} 43$, which has been previously demonstrated to be an essential element in the protective response of the myocardium to ischemic preconditioning (19).
To date, there are fewer reports on AQPs and their relationship with connexins in the heart when compared to the kidney, brain, eye, and other tissues. The function of AQPs and connexins in the heart is poorly understood, and there is 
considerable controversy among different studies. AQP1 has been identified in rodent heart and human heart (20), and it has been confirmed that AQP1 is the dominant AQP in the human heart and that it is co-localized with t-tubular and caveolar proteins, in particular.

It has been hypothesized that the transmembrane ion current of myocardial cells, mediated by AQP1, requires rapid equilibrium of internal and external osmotic pressure $(21,22)$. In a previous report, it was demonstrated that AQP1 expression was increased in anemic fetuses compared to agematched controls, suggesting that AQP1 plays an important role in physiological accommodation to fetal anemia (23). In this study, left ventricular mRNA and protein expression levels of AQP1 were measured following CPB surgery and the water content of myocardial muscle was determined. Our results indicated that cardiac AQP1 plays a role during the osmotic stress of CPB and the osmotic stress that occurs following cardiac edema.

It was previous demonstrated that AQP1 is present in the pulmonary microvascular endothelium, but on the expression of AQP1 decreases in alveolar microvessels upon pulmonary edema in chronic heart failure. It has been hypothesized that downregulation of AQP1 in alveolar microvessels potentially acts as a compensatory mechanism to protect against the formation of excessive pulmonary edema (24). The expression of AQP1 in alveolar microvessels following edema was not observed in this study. This was most likely because AQP proteins in myocardial cells are the major water transport channels; moreover, $\mathrm{Hg}^{2+}$ (AQP protein non-specific inhibitor) limits the extent of myocardial edema. We found no statistically significant differences between mRNA and protein expression levels of AQP1 following $\mathrm{Hg}^{2+}$ treatment during CPB surgery. However, the water content of myocardial muscle tissue significantly decreased after $\mathrm{Hg}^{2+}$ treatment.

In this study, we analyzed the expression level of the main gap junction protein, $\mathrm{Cx} 43$; we hypothesized that there is a potential relationship between connexins and aquaporins. Goats with a silenced AQP1 gene exhibit increased Cx43 levels following CPB surgery, suggesting a close relationship between AQP1 and Cx43.

In summary, we found that $\mathrm{AQP} 1$ plays a role in myocardial edema following CPB surgery; moreover, a close relationship between AQP1 and Cx43 was demonstrated. Further studies are warranted to investigate potential methods of regulating AQP1 expression in order to control the degree of myocardial edema during surgery.

\section{Acknowledgements}

This study was partly supported by grants from the National Natural Science Foundation of China (30872558) and the Science and Technology Foundation of School of Medicine, Shanghai Jiaotong University (2008XJ019).

\section{References}

1. Albers J, Schroeder A, de Simone R, Mockel R, Vahl CF and Hagl S: 3D evaluation of myocardial edema: experimental study on 22 pigs using magnetic resonance and tissue analysis. Thorac Cardiovasc Surg 49: 199-203, 2001.
2. Jia CX, Rabkin DG, Hart JP, Dean DA, Cabreriza SA, Weinberg AD and Spotnitz HM: Regional variation in myocardial water content in the edematous pig heart. J Surg Res 106: 70-75, 2002.

3. Foglia RP, Lazar HL, Steed DL, Follette DM, Manganaro AJ, Deland E and Buckberg GD: Iatrogenic myocardial edema with crystalloid primes: effects on left ventricular compliance, performance, and perfusion. Surg Forum 29: 312-315, 1978.

4. Laine GA and Allen SJ: Left ventricular myocardial edema. Lymph flow, interstitial fibrosis, and cardiac function. Circ Res 68: 1713-1721, 1991.

5. Spotnitz HM and Hsu DT: Myocardial edema: importance in the study of left ventricular function. Adv Card Surg 5: 1-25, 1994.

6. Palmer BS, Hadziahmetovic M, Veci T and Angelos MG: Global ischemic duration and reperfusion function in the isolated perfused rat heart. Resuscitation 62: 97-106, 2004.

7. Verkman AS: Physiological importance of aquaporins: lessons from knockout mice. Curr Opin Nephrol Hypertens 9: 517-522, 2000.

8. Verkman AS: Aquaporin water channels and endothelial cell function. J Anat 200: 617-627, 2002.

9. King LS, Kozono D and Agre P: From structure to disease: the evolving tale of aquaporin biology. Nat Rev Mol Cell Biol 5: 687-698, 2004.

10. Page E, Winterfield J, Goings G, Bastawrous A and UpshawEarley J: Water channel proteins in rat cardiac myocyte caveolae: osmolarity-dependent reversible internalization. Am J Physiol 274: H1988-H2000, 1998.

11. Umenishi F, Verkman AS and Gropper MA: Quantitative analysis of aquaporin mRNA expression in rat tissues by RNase protection assay. DNA Cell Biol 15: 475-480, 1996.

12. Nielsen S, Smith BL, Christensen EI and Agre P: Distribution of the aquaporin CHIP in secretory and resorptive epithelia and capillary endothelia. Proc Natl Acad Sci USA 90: 7275-7279, 1993.

13. Boengler K, Dodoni G, Rodriguez-Sinovas A, Cabestrero A, Ruiz-Meana M, Gres P, Konietzka I, Lopez-Iglesias C, GarciaDorado D, Di Lisa F, et al: Connexin 43 in cardiomyocyte mitochondria and its increase by ischemic preconditioning. Cardiovasc Res 67: 234-244, 2005.

14. Gorbe A, Varga ZV, Kupai K, Bencsik P, Kocsis GF, Csont T, Boengler K, Schulz R and Ferdinandy P: Cholesterol diet leads to attenuation of ischemic preconditioning-induced cardiac protection: the role of connexin 43. Am J Physiol Heart Circ Physiol 300: H1907-H1913, 2011.

15. Ruiz-Meana M, Rodriguez-Sinovas A, Cabestrero A, Boengler K, Heusch G and Garcia-Dorado D: Mitochondrial connexin43 as a new player in the pathophysiology of myocardial ischaemiareperfusion injury. Cardiovasc Res 77: 325-333, 2008.

16. Gross C, Nakamoto M, Yao X, Chan CB, Yim SY, Ye K, Warren ST and Bassell GJ: Excess phosphoinositide 3-kinase subunit synthesis and activity as a novel therapeutic target in fragile X syndrome. J Neurosci 30: 10624-10638, 2010.

17. Parsa CJ, Matsumoto A, Kim J, Riel RU, Pascal LS, Walton GB, Thompson RB, Petrofski JA, Annex BH, Stamler JS, et al: A novel protective effect of erythropoietin in the infarcted heart. J Clin Invest 112: 999-1007, 2003.

18. Egan JR, Butler TL, Au CG, Tan YM, North KN and Winlaw DS: Myocardial water handling and the role of aquaporins. Biochim Biophys Acta 1758: 1043-1052, 2006.

19. Garcia-Dorado D, Ruiz-Meana M, Padilla F, RodriguezSinovas A and Mirabet M: Gap junction-mediated intercellular communication in ischemic preconditioning. Cardiovasc Res 55: 456-465, 2002.

20. Butler TL, Au CG, Yang B, Egan JR, Tan YM, Hardeman EC, North KN, Verkman AS and Winlaw DS: Cardiac aquaporin expression in humans, rats, and mice. Am J Physiol Heart Circ Physiol 291: H705-H713, 2006.

21. Au CG, Cooper ST, Lo HP, Compton AG, Yang N, Wintour EM, North KN and Winlaw DS: Expression of aquaporin 1 in human cardiac and skeletal muscle. J Mol Cell Cardiol 36: 655-662, 2004.

22. Suleymanian MA and Baumgarten CM: Osmotic gradientinduced water permeation across the sarcolemma of rabbit ventricular myocytes. J Gen Physiol 107: 503-514, 1996.

23. Jonker S, Davis LE, van der Bilt JD, Hadder B, Hohimer AR, Giraud GD and Thornburg KL: Anaemia stimulates aquaporin 1 expression in the fetal sheep heart. Exp Physiol 88: 691-698, 2003.

24. Mullertz KM, Strom C, Trautner S, Amtorp O, Nielsen S, Christensen S, Haunso S and Jonassen TE: Downregulation of aquaporin-1 in alveolar microvessels in lungs adapted to chronic heart failure. Lung 189: 157-166, 2011. 